\title{
The effect of G-FAST (Gaze, Face, Arm, Speech, and Time) training on emergency medical technicians for identifying patients with large vessel occlusion in Taipei.
}

Yu-Chen Chiua; Ming-Ju Hsiehb; Jen-Tang, Sun ba*; Yu-Chun Chiend; Yao Cheng Wangd; Yueh-Ping Liü; Matthew Huei-Ming Ma ${ }^{\mathrm{b}, \mathrm{c}}$; Wen-Chu Chiang ${ }^{\mathrm{b}, \mathrm{c}}$

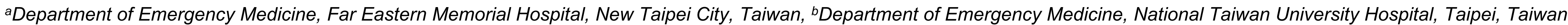
cDepartment of Emergency Medicine, National Taiwan University Hospital, Yun-Lin Branch, Yun-Lin County, Taiwan, dc Fire Department, Taipei City, Taiwan

\section{Background}

Endovascular thrombectomy has been proven to benefit patients with large vessel occlusion (LVO). The sooner the endovascular treatment is given, the better the functional outcome. Therefore, resuscitation guidelines recommended the strategy of bypassing the patients with LVO to the hospitals with endovascular capabilities. However, in the past, there was no training course for emergency medical technicians (EMTs) to identify patients with LVO in Taiwan.

\section{Objective}

We evaluated the effect of the training course on EMTs for identifying patients with LVO by G-FAST score (Gaze, Face, Arm, Speech, and Time). The effect was measured by the changes in knowledge, attitude, confidence, and simulation performance of trainees.

Figure 1 The field stroke score of Taipei City
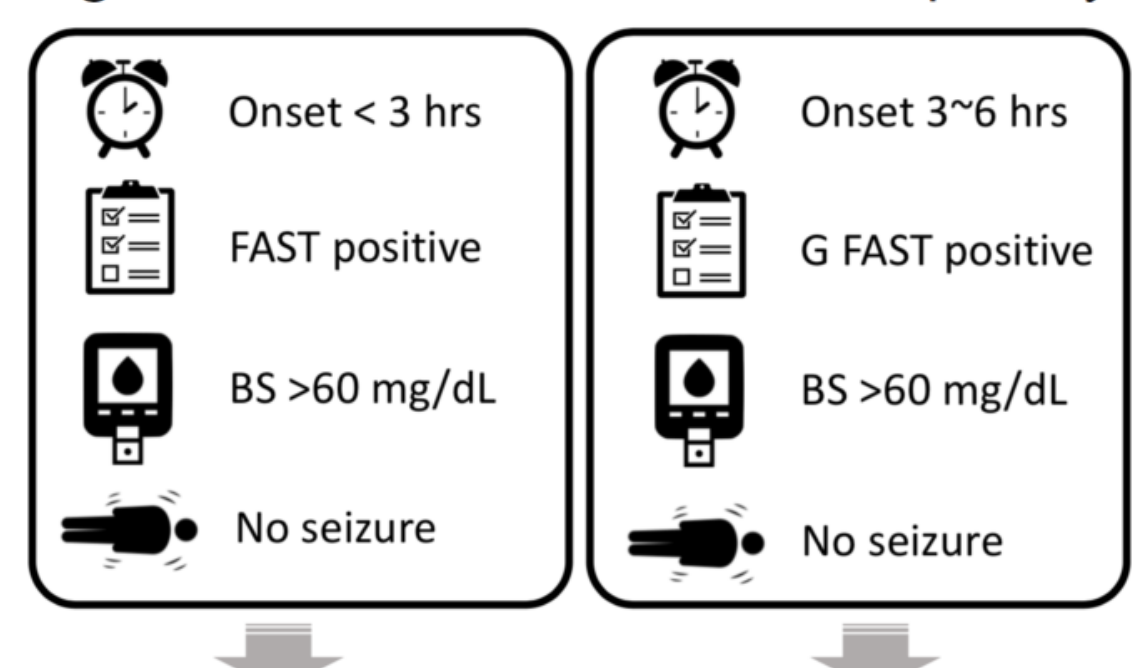

tPA center

A center

\section{Method}

EMTs in Taipei City were enrolled in the G-FAST training course from May 15th to November 15th, 2017. The course included a 30-minute lecture and a 20-minute video of performing GFAST.

Figure 2 The algorithm of this study

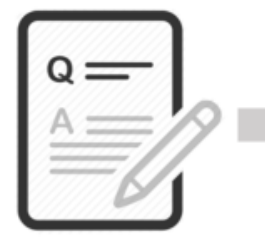

Pre-test

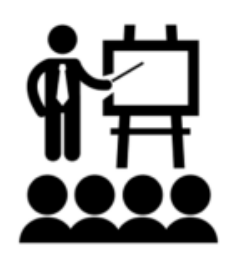

$30 \mathrm{~min}$ lecture

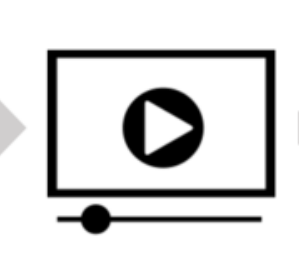

$20 \mathrm{~min}$ video

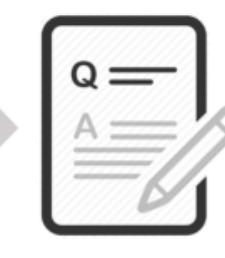

Post-test
The trainees received exams with the same questions before and after the course. The exams included: (1) the questionnaire regarding knowledge (full score, 10), attitude and confidence (using 5-point Likert scales) of providing care to patients with stroke, and (2) simulation tests by watching ten videos and answering the questions about scenario judgment, including the parameters of G-FAST and the decision of destination hospital.

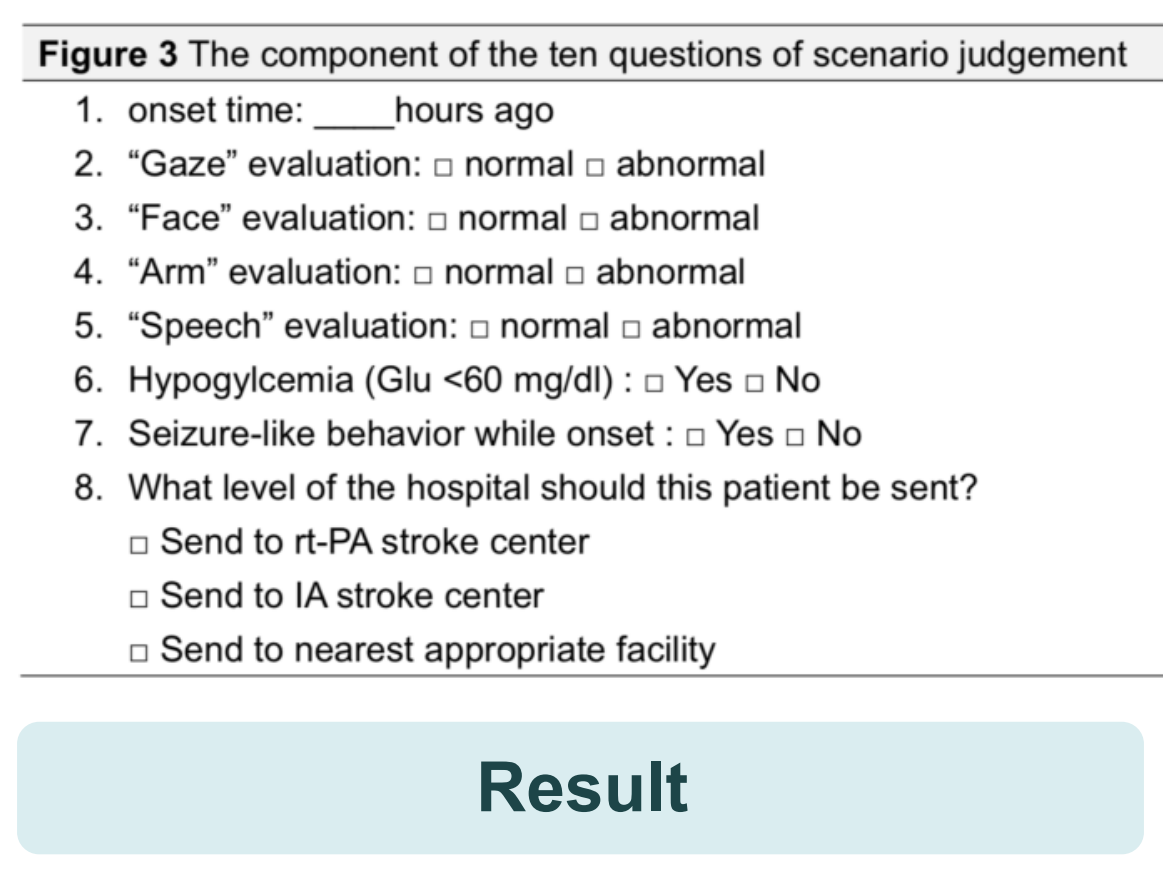

Totally 1058 EMTs were enrolled. Significant improvement was found in knowledge (8.80 vs 9.36, $\mathrm{p}<0.001$ ) attitude (all items, $\mathrm{p}<0.001$ ), confidence (all items, $\mathrm{p}<0.001$ ), and choosing the appropriate destination hospitals ( 5.48 vs 7.83, $\mathrm{p}<0.001)$ in simulation test after training. Among participants who chose the inappropriate hospitals, they tended to transport patients to hospitals of higher level despite they correctly judged the G-FAST parameters.

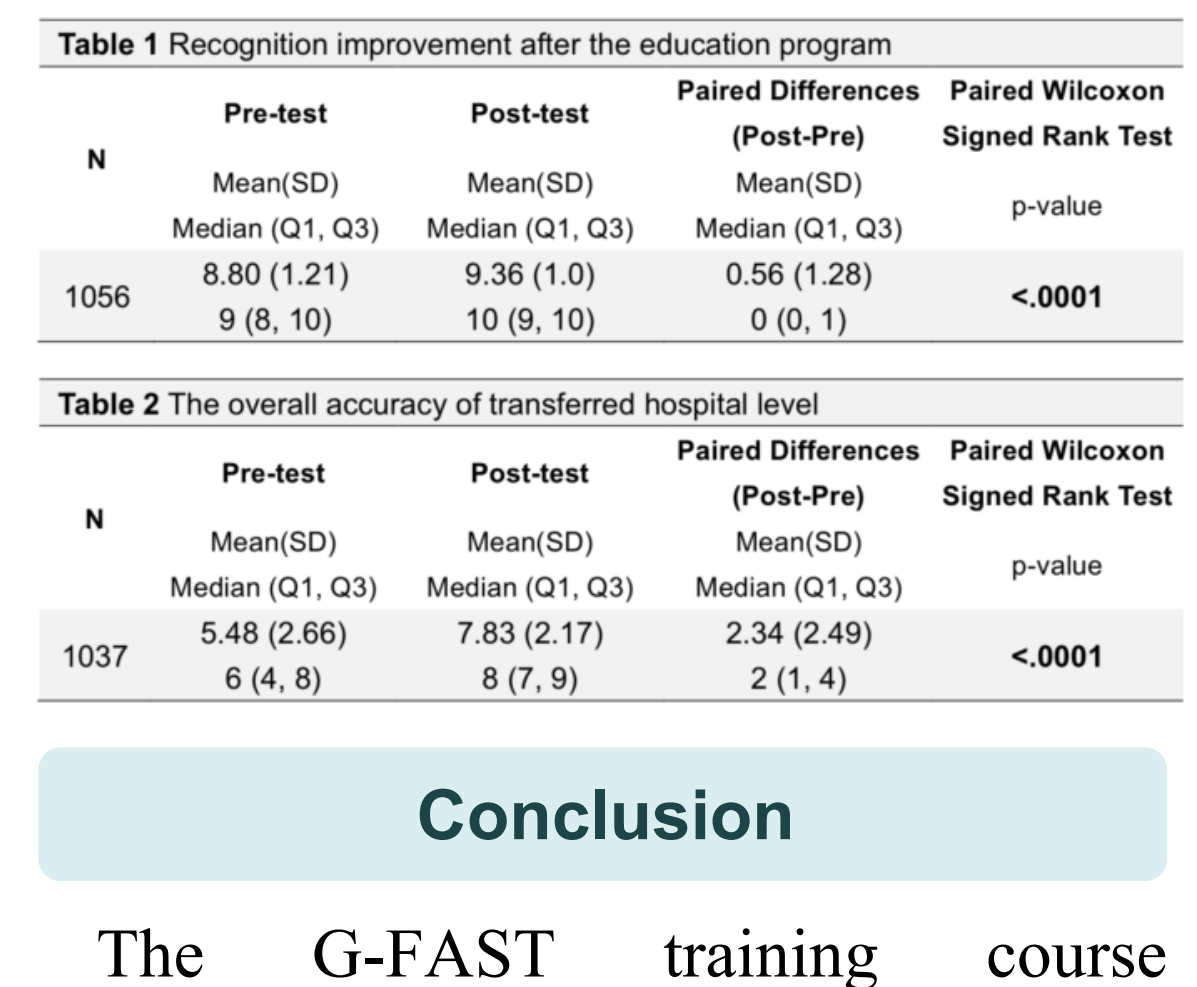

The G-FAST training course significantly improved the knowledge, attitude, and confidence and simulation performance of EMTs in identifying patients with LVO in Taiwan. EMTs tended to transport patients to higherlevel hospitals than their judgment. FAR EASTERN MEMORIAL HOSPITAL
臺北市政府消防局 TAIPEI CITY FIRE DEPARTMENT 